\title{
Working Memory Contributions to Reinforcement Learning Impairments in Schizophrenia
}

\author{
Anne G.E. Collins, ${ }^{1}$ Jaime K. Brown, ${ }^{2}$ James M. Gold, ${ }^{2}$ James A. Waltz, ${ }^{2}$ and ${ }^{\oplus}$ Michael J. Frank ${ }^{1}$ \\ ${ }^{1}$ Department of Cognitive, Linguistics, and Psychological Sciences, Brown University, Providence, Rhode Island 02912, and ${ }^{2}$ Maryland Psychiatric Research \\ Center, Department of Psychiatry, University of Maryland School of Medicine, Baltimore, Maryland 21201
}

\begin{abstract}
Previous research has shown that patients with schizophrenia are impaired in reinforcement learning tasks. However, behavioral learning curves in such tasks originate from the interaction of multiple neural processes, including the basal ganglia- and dopaminedependent reinforcement learning (RL) system, but also prefrontal cortex-dependent cognitive strategies involving working memory (WM). Thus, it is unclear which specific system induces impairments in schizophrenia. We recently developed a task and computational model allowing us to separately assess the roles of RL (slow, cumulative learning) mechanisms versus WM (fast but capacity-limited) mechanisms in healthy adult human subjects. Here, we used this task to assess patients' specific sources of impairments in learning. In 15 separate blocks, subjects learned to pick one of three actions for stimuli. The number of stimuli to learn in each block varied from two to six, allowing us to separate influences of capacity-limited WM from the incremental RL system. As expected, both patients $(n=49)$ and healthy controls $(n=36)$ showed effects of set size and delay between stimulus repetitions, confirming the presence of working memory effects. Patients performed significantly worse than controls overall, but computational model fits and behavioral analyses indicate that these deficits could be entirely accounted for by changes in WM parameters (capacity and reliability), whereas RL processes were spared. These results suggest that the working memory system contributes strongly to learning impairments in schizophrenia.
\end{abstract}

Key words: computational model; reinforcement learning; schizophrenia; working memory

\section{Introduction}

Patients with schizophrenia (SZ) demonstrate deficits across a wide range of measures of executive control/working memory (WM; Barch and Ceaser, 2012) and reinforcement learning (RL; Gold et al., 2008; Deserno et al., 2013) paradigms, including the Iowa Gambling Task (Shurman et al., 2005), the Wisconsin Card Sorting Test (Prentice et al., 2008), probabilistic reinforcement, and reversal learning (Waltz and Gold, 2007; Waltz et al., 2007, 2011; Schlagenhauf et al., 2013). However, the specific underlying cognitive and neural mechanisms remain uncertain: most tasks involve multiple cognitive and neural processes, including the striatal dopamine system, which is involved in signaling prediction errors and integrating them over trials (Frank et al., 2004; Dayan and Daw, 2008), and the prefrontal cortex, which is involved in using WM to test hypotheses and represent values of prospective outcomes to guide choice.

Patients with SZ consistently exhibit deficits in tasks involving learning (Paulus et al., 2003; Kim et al., 2007), but a better understanding of these deficits necessitates parsing the contribution of

\footnotetext{
Received March 9, 2014; revised Aug. 25, 2014; accepted Aug. 28, 2014

Author contributions: A.G.E.C., J.M.G., J.A.W., and M.J.F. designed research; A.G.E.C. and J.K.B. performed research; A.G.E.C. analyzed data; A.G.E.C., J.M.G., J.A.W., and M.J.F. wrote the paper.

This work was supported by NIMH Grant R01 MH080066-01. We gratefully acknowledge the efforts of Leeka Hubzin and Sharon August in data collection.

The authors declare no competing financial interests.

Correspondence should be addressed to Anne G.E. Collins at the above address. E-mail: anne_collins@brown.edu.

DOI:10.1523/JNEUROSCI.0989-14.2014

Copyright $\odot 2014$ the authors $\quad 0270-6474 / 14 / 3413747-10 \$ 15.00 / 0$
}

different neurocognitive processes. Since most learning paradigms confound contributions of two separable cognitive processes, WM and RL, it remains unclear whether patient impairments arise from core changes in the RL process per se (where "RL" is used to signify the incremental accumulation of values based on reward prediction errors).

Computational models of RL afford trial-by-trial quantification of choices (O'Doherty et al., 2007; Daw, 2011) and are thought to reflect dopamine-mediated corticostriatal synaptic plasticity (Frank et al., 2007). However, computational modeling by itself does not solve the problem of task impurity: other cognitive functions involved in RL tasks (such as working memory) may contaminate the inferred RL processes.

Collins and Frank (2012) developed a task to disentangle WM and RL contributions to learning by explicitly manipulating potential WM load. They also presented a hybrid model that included a model-free RL process, reflecting the striatumdopaminergic system; and a capacity-limited, decay-sensitive WM process. They showed that classical RL models overestimated learning rates but that the hybrid model was able to separately estimate the contribution of WM from RL. Furthermore, once WM processes were controlled, individual differences in the RL learning rate were related to genetic variants in GPR6, a protein selectively expressed in the striatum (Lobo et al., 2007). Conversely, WM capacity estimates were related to COMT, a gene that preferentially affects prefrontal function (Gogos et al., 1998; Huotari et al., 2002; Matsumoto et al., 2003; Frank et al., 2007; Slifstein et al., 2008). 
Table 1. Demographics and clinical characteristics of patients with schizophrenia and healthy control comparison participants

\begin{tabular}{|c|c|c|c|c|c|}
\hline & \multicolumn{2}{|c|}{ Schizophrenia $(n=49)$} & \multicolumn{2}{|c|}{ Healthy control $(n=36)$} & \multirow[b]{2}{*}{ Group comparison } \\
\hline & Mean & (SD) & Mean & (SD) & \\
\hline \multicolumn{6}{|l|}{ Demographics } \\
\hline Age & 39.46 & $(10.66)$ & 41.36 & $(9.85)$ & $F=0.7 ; p=0.40$ \\
\hline Gender (\% males) & 71 & & 69 & & $\chi^{2}(1, n=85)=0.0 ; p=0.84$ \\
\hline Race and ethnicity (\% Cauc:\% AA:\% other) & $53: 35: 12$ & & $61: 31: 8$ & & $\chi^{2}(5, n=85)=2.0 ; p=0.85$ \\
\hline Personal education & 12.73 & (2.16) & 15.17 & (2.01) & $F=27.9 ; p<0.001$ \\
\hline Mother's education & 14.00 & (2.79) & 13.83 & (2.55) & $F=0.1 ; p=0.78$ \\
\hline Father's education & $14.86^{a}$ & (3.11) & $14.41^{b}$ & (3.19) & $F=0.4 ; p=0.54$ \\
\hline \multicolumn{6}{|l|}{ Neuropsychology measures } \\
\hline WASI & 101.20 & $(14.72)$ & 118.26 & (11.53) & $F=34.1 ; p<0.001$ \\
\hline WRAT4 & 95.47 & (14.63) & 111.03 & (11.50) & $F=24.8 ; p<0.001$ \\
\hline WTAR & 97.51 & (17.26) & 111.67 & (10.90) & $F=18.7 ; p<0.001$ \\
\hline MCCB & 32.94 & (13.76) & 54.14 & (10.47) & $F=59.8 ; p<0.001$ \\
\hline \multicolumn{6}{|l|}{ Clinical variables } \\
\hline SANS total & 25.91 & $(13.41)$ & & & \\
\hline BNSS total & 21.33 & (14.11) & & & \\
\hline BPRS total & 32.20 & $(6.21)$ & & & \\
\hline LOF total & 21.9 & (5.81) & & & \\
\hline IIIness duration (years) & 19.6 & (11.4) & & & \\
\hline
\end{tabular}

Cauc, Caucasian; AA, African American; WASI, Wechsler Abbreviated Scale of Intelligence; WRAT, Wide Range Achievement Test Reading; WTAR, Wechsler Test of Adult Reading; LOF: Level of Function.

${ }^{a} n=43$.

${ }^{b} n=34$.

We apply a similar experimental and modeling strategy to investigate whether learning impairments in SZ patients are more closely related to WM processes or to core aspects of the RL system. Based on recent studies (Waltz and Gold, 2007; Strauss et al., 2011; Gold et al., 2012; Doll et al., 2014), we hypothesized that patients would exhibit deficits in WM processes with relative sparing of incremental, model-free RL processes (Waltz et al., 2009; Dowd and Barch, 2012).

\section{Materials and Methods \\ Patient-related methods}

A total of 49 people ( 35 males and 14 females) with a DSM-IV (American Psychiatric Association, 2000) diagnosis of schizophrenia $(n=44)$ or schizoaffective disorder $(n=5)$ participated. All were clinically stable outpatients recruited from the MPRC Outpatient Research Program or from other nearby outpatient clinics. Diagnosis was determined by the Structured Clinical Interview for DSM-IV Axis I Disorders (SCID; First et al., 1997), past medical records, and clinician reports. At the time of testing, all participants had been on stable medications (same type and dose) for a minimum of 4 weeks. Duration of illness was also recorded.

A total of 36 healthy volunteers (25 males and 11 females), matched to the patient group in terms of important demographic variables (Table 1), were recruited through a combination of random telephone number dialing, Internet advertisements, and word of mouth among recruited controls. All were screened with the SCID and the Structured Clinical Interview for DSM-IV Personality Disorders (First et al., 1997; Pfohl et al., 1997) and were free of a lifetime history of psychosis, current Axis I disorder, and Axis II schizophrenia spectrum disorders. All denied a family history of psychosis in first-degree relatives. All control participants denied a history of medical or neurological disease, including current or recent substance abuse or dependence that would likely impact cognitive performance.

All participants received the Wechsler Abbreviated Scale of Intelligence (Wechsler, 1999) and the MATRICS Consensus Cognitive Battery (MCCB; Nuechterlein and Green, 2006) to assess the overall level of cognitive ability, as well as a measure of working memory performance that we used in correlational analyses with model-based estimates of WM. Participants with SZ also received the Scale for the Assessment of Negative Symptoms (SANS; Andreasen, 1983), the Brief Negative Symptom Rating Scale (BNSS; Kirkpatrick et al., 2011), and the Brief Psychiatric Rating Scale (BPRS; Overall and Gorham, 1962) to assess symptom severity. The patients were mild-moderately symptomatic as seen on the clinical rating scales. The healthy controls scored significantly higher on measures of word reading, estimated IQ, and general neuropsychological ability as is typical in the literature.

\section{Experimental design}

The task was modified from a classic conditional associative learning paradigm (Petrides, 1985; Fig. 1) in which, on each trial, a single stimulus was presented to which subjects could respond with one of three responses (button presses on a response pad). Subjects had to learn over time which of those responses was the correct one to select for each stimulus, based on the binary deterministic feedback they received (Collins and Frank, 2012).

To manipulate working memory demands separately from RL components, we systematically varied the number of stimuli (denoted as set size $n_{\mathrm{s}}$ ) for which subjects had to learn the correct actions within a block. There were four blocks with $n_{\mathrm{S}}=2$, two blocks each with $n_{\mathrm{S}}=3$ or $n_{\mathrm{S}}=$ 4 , three blocks with $n_{\mathrm{S}}=5$, and two blocks with $n_{\mathrm{S}}=6$ for a total of 13 blocks, requiring $\sim 35 \mathrm{~min}$ to complete. The number of blocks per set size was determined by a trade-off between three constraints: equating the overall number of stimuli per set size, obtaining sufficient blocks of higher set size to ensure taxing of the RL system, and the overall experiment length. Each block corresponded to a different category of visual stimulus (such as sports, fruits, places, etc.), with stimulus category assignment to block set size counterbalanced across subjects. Block ordering was also counterbalanced within subjects to ensure an even distribution of high/low load blocks across the duration of the experiment.

At the beginning of each block, subjects were shown the entire set of stimuli for that block and encouraged to familiarize themselves with them. They were then asked to answer as quickly and accurately as possible after each individual stimulus presentation. Within each block, stimuli were presented in a pseudo-randomly intermixed order, with a minimum of 9 and a maximum of 15 presentations of each stimulus, up to a performance criterion of at least four correct responses of the five last presentations of each stimulus.

Stimuli were presented in the center of the screen for up to $3.5 \mathrm{~s}$, during which time subjects could press one of three keys. Binary deterministic auditory feedback ensued (high tone for correct and low tone for incorrect, as well as the words "correct" or "incorrect," presented for $1 \mathrm{~s}$ ). A $0.75 \mathrm{~s}$ dark screen followed this, before the next stimulus.

Subjects were instructed that finding the correct action for one stimulus was not informative about the correct action for another stimulus. This was enforced in the choice of correct actions, such that, in a block 


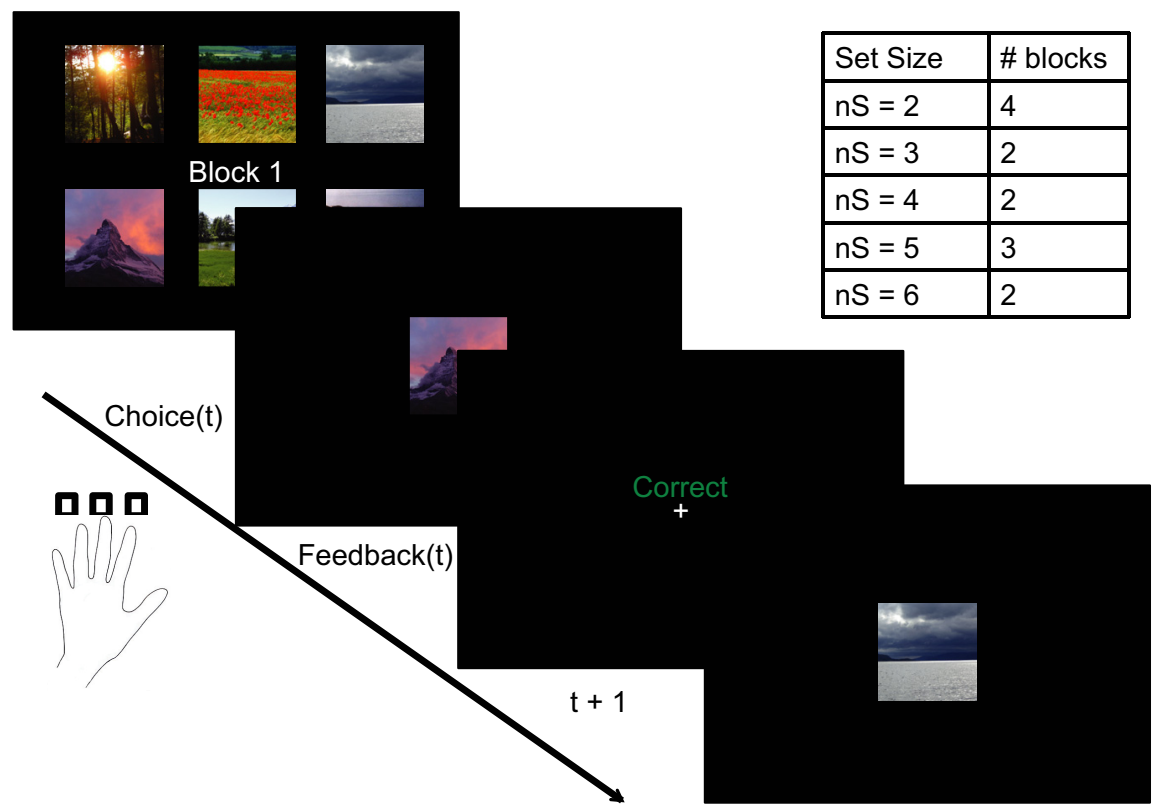

Figure 1. Experimental protocol. At the beginning of each block, subjects were presented the set of stimuli they would have to learn the correct actions for in that block. Each trial included a $3.5 \mathrm{~s}$ presentation of a stimulus during which time subjects pressed one of three responses. Feedback indicating correct or incorrect followed. Block set sizes varied between two and six, and the order was randomized across subjects.

with $n_{\mathrm{S}}=3$, for example, the correct actions for the three stimuli were not necessarily three distinct keys. This procedure was implemented to ensure independent learning of all stimuli (i.e., to prevent subjects from inferring the correct actions to stimuli based on knowing the actions for other stimuli).

\section{Computational modeling \\ RLWM model}

To better account for subjects' behavior and disentangle roles of working memory and reinforcement learning, we fitted subjects' choices with our RLWM computational model. Previous research showed that a model allowing choice to be a mixture between a classic $\delta$ rule reinforcement learning process and a fast capacity-limited working memory process accounted best for learning (Collins and Frank, 2012). The model used here is a variant of the previously published model. We first summarize its key properties.

(1) RLWM includes two modules, a classic incremental RL module with learning rate $\alpha$ and a WM module that can learn in a single trial (learning rate 1) but is capacity limited (with capacity $K$ ).

(2) Both RL and WM modules are subject to forgetting [decay parameters $\phi_{\mathrm{RL}}$ and $\phi_{\mathrm{WM}}$ and noise in the choice policy (directed: softmax $\beta$, undirected: $\varepsilon$ )]. We also include a perseveration parameter pers to account for potential tendencies to repeat actions in the face of negative feedback.

(3) The final action choice is controlled by weighing the contributions of the RL and WM modules' policies. How much weight is given to WM relative to RL (the mixture parameter) depends on two factors. First, it depends on what the probability is that a stimulus is stored in WM of capacity $K$. If there are fewer stimuli than WM can hold $\left(n_{\mathrm{S}} \leq K\right)$, then that probability is 1. Otherwise, only $K$ out of $n_{\mathrm{S}}$ can be stored. Second, the overall reliance of WM versus $\mathrm{RL}$ is scaled by factor $0<\rho<1$, with higher values reflecting relative greater confidence in $\mathrm{WM}$ function. Thus, the weight given to the WM policy relative to the RL policy is $w=\rho \times \min (1$, $\left.K / n_{\mathrm{S}}\right)$.

We conducted extensive comparisons of multiple models to determine which fit the data best (penalizing for complexity) so as to validate the use of this model in interpreting subjects' data. In particular, we fit several other models that include or leave out various properties of the RLWM model.

\section{Learning model details}

Reinforcement learning model. All models include a standard RL module with simple $\delta$ rule learning. For each stimulus $s$ and action $a$, the expected reward $Q(s, a)$ is learned as a function of reinforcement history. Specifically, the $Q$ value for the selected action given the stimulus is updated after observing each trial's reward outcome $r_{\mathrm{t}}$ ( 1 for correct, 0 for incorrect) as a function of the prediction error between expected and observed reward at trial $t$, as follows:

$$
Q_{\mathrm{t}+1}(s, a)=Q_{\mathrm{t}}(s, a)+\alpha \times \delta_{\mathrm{t}},
$$

where $\delta_{\mathrm{t}}=r_{\mathrm{t}}-Q_{\mathrm{t}}(s, a)$ is the prediction error and $\alpha$ is the learning rate. Choices are generated probabilistically with greater likelihood of selecting actions that have higher $Q$ values, using the following softmax choice rule:

$$
p(a \mid s)=\exp (\beta Q(s, a)) / \Sigma_{\mathrm{i}}\left(\exp \left(\beta Q\left(s, a_{\mathrm{i}}\right)\right)\right) .
$$

Here, $\beta$ is an inverse temperature determining the degree with which differences in $Q$ values are translated into a more deterministic choice and the sum is over the three possible actions $a_{\mathrm{i}}$

Undirected noise. The softmax temperature allows for stochasticity in choice in an oriented way, by making more valuable actions more likely. We also allow for "slips" of action ("irreducible noise," i.e., even when $Q$ value differences are large). Given a model's policy $\pi=p(a \mid s)$, adding undirected noise consists of defining the new mixture policy as follows:

$$
\pi^{\prime}=(1-\varepsilon) \pi+\varepsilon U,
$$

where $U$ is the uniform random policy $\left(U(a)=1 / n_{\mathrm{A}}, n_{\mathrm{A}}=3\right)$ and the parameter $0<\varepsilon<1$ controls the amount of noise (Collins and Koechlin, 2012; Guitart-Masip et al., 2012; Collins and Frank, 2013).

Forgetting. We allow for potential decay or forgetting in $Q$ values on each trial, additionally updating all $Q$ values at each trial, according to the following:

$$
Q \leftarrow Q+\phi\left(Q_{0}-Q\right),
$$

where $0<\phi<1$ is a decay parameter pulling at each trial the estimates of values toward initial value $Q_{0}=1 / n_{\mathrm{A}}$

Perseveration. Previous studies have shown differential treatment of positive and negative outcomes in patients in probabilistic learning tasks, as well as perseveration (neglect of negative outcomes) in adaptive cognitive tasks such as the Wisconsin Card Sorting Test (Kim et al., 2007; Prentice et al., 2008). To allow for potential neglect of negative, as opposed to positive, feedback, we estimate a perseveration parameter pers such that for negative prediction errors $(\delta<0)$, the learning rate $\alpha$ is reduced by $\alpha=(1-$ pers $) \times \alpha$. Thus, values of pers near 1 indicate perseveration with complete neglect of negative feedback, whereas values near 0 indicate equal learning from negative and positive feedback. Note that this notion of perseveration is focused on the failure to use negative feedback to avoid repeating an error, rather than to shift away from a previously reinforced option, as is often implied in the Wisconsin Card Sorting Test (Kim et al., 2007; Prentice et al., 2008).

Working memory. To implement an approximation of a rapid updating but capacity-limited WM, this feature assumes a learning rate $\alpha=1$ but includes capacity limitation such that only, at most, $K$ stimuli can be remembered. At any time, the probability of a given stimulus being in working memory is $p_{\mathrm{WM}}=\rho \times \min \left(1, K / n_{\mathrm{S}}\right)$. As such, the overall policy is as follows: 


$$
\pi=p_{\mathrm{WM}} \pi_{\mathrm{WM}}+\left(1-p_{\mathrm{WM}}\right) \pi_{\mathrm{other}}
$$

where $\pi_{\mathrm{WM}}$ is the WM softmax policy and $\pi_{\mathrm{other}}$ is another module's policy. In the WM-only model, this is the random uniform policy: $\pi_{\text {other }}$ $=U$. In the RLWM model, this is $\pi_{\mathrm{other}}=\pi_{\mathrm{RL}}$ : the softmax policy from the RL module without capacity limitation. Note that this implementation assumes that information stored for each stimulus in working memory pertains to action-outcome associations. Furthermore, this implementation is an approximation of a capacity-/resource-limited notion of working memory. It captures key aspects of working memory such as (1) rapid and accurate encoding of information when a low amount of information is to be stored and (2) decrease in the likelihood of storing or maintaining items when more information is presented or when distractors are presented during the maintenance period. Because it is a probabilistic model of WM, it cannot capture specifically which items are stored, but it can provide the likelihood of storing and maintaining items given task structure (set size, delay, etc.).

\section{Models considered}

We combined the previously described features into different learning models and investigated which ones provided the best fit of subjects' data. For all models we considered, adding undirected noise, forgetting, and perseveration features significantly improved the fit, accounting for added model complexity (see model comparisons).

This left three interesting classes of models to consider.

(1) RL: This model combines simple $\delta$ rule RL, with forgetting, perseveration, and undirected noise features. It assumes a single system that is sensitive to delay and asymmetry in feedback processing. This is a five-parameter model (learning rate $\alpha$, sofmax inverse temperature $\beta$, undirected noise $\varepsilon$, decay $\phi_{\mathrm{RL}}$, and pers parameter).

(2) RLWM: This is the main model, consisting of a mixture of RL and working memory. RL and WM modules have shared softmax $\beta$ and pers parameters but separate decay parameters, $\phi_{\mathrm{RL}}$ and $\phi_{\mathrm{WM}}$. Working memory capacity is $0<K<6$, with reliance $0<\rho<1$ on working memory for items potentially stored in working memory. Undirected noise is added to the RLWM mixture policy. This is an eight-parameter model (capacity $K$, WM reliance $\rho$, WM decay $\phi_{\mathrm{WM}}$, RL learning rate $\alpha$, RL decay $\phi_{\mathrm{RL}}$, perseveration pers, sofmax inverse temperature $\beta$, undirected noise $\varepsilon$ ).

(3) WM: This is the WM-only model. It is equivalent to the previous one with RL learning rate $\alpha=0$, RL decay $\phi_{\mathrm{RL}}=0$. It assumes that any choice not made by working memory is made randomly (uniform). It is a six-parameter model.

\section{RLWM fitting procedure}

Parameters were fit using Matlab optimization under the constraint function fmincon. This was iterated with 50 randomly chosen starting points to increase the likelihood of finding a global rather than local optimum. For models including the discrete capacity parameter, this fitting was performed iteratively for capacities $n=\{0,1,2,3,4,5,6\}$, then inferring capacity and other parameters that gave the best fit.

Softmax $\beta$ temperature was fit with constraints [0 500]. All other parameters were fit with constraints $\left[\begin{array}{ll}0 & 1\end{array}\right]$. We considered sigmoidtransforming the parameters to avoid constraints in optimization and obtain normal distributions, but whereas fit results were similar, distributions obtained were actually not normal. Thus, all statistical tests on parameters were nonparametric.

\section{Model comparison}

We used the Akaike Information Criterion (AIC) to penalize model complexity (Burnham and Anderson, 2002). Indeed, we previously showed that AIC was a better approximation than the Bayesian Information Criterion (Schwarz, 1978) at recovering the true model from generative simulations of the WMRL model and variants (Collins and Frank, 2012). We used the Matlab spm_bms function to compute Bayesian model evidence over AIC (Stephan et al., 2009) for Bayesian model selection over the group. Models WM-only and RL-only were strongly nonfa- vored, with probability 0 over the whole group. This was also true when investigating separately each group, with evidence in favor of RLWM > 0.97 .

\section{Results}

Data were successfully collected for 49 patients (SZ) and 36 healthy controls (HCs). All subjects reached asymptotic performance above chance in set sizes of two to three. Although three patients did not show improvement for higher set sizes, we chose to not remove any subjects from the analysis as they did learn in lower set sizes. Exclusion of these three subjects did not change the effects of interests, however.

We first investigated learning curves per stimulus in different set sizes. These analyses (Fig. 2, top) revealed that both healthy controls and patients learned to select the correct action for each stimulus in all set sizes and that both groups showed an effect of set size. Importantly, learning slowed when there were more stimuli to be learned in parallel, with healthy controls reaching asymptotic performance by iteration 3 in set size two (corresponding to "perfect memory" performance).

To compare learning performance across groups and conditions, we first entered average performance in a multiple regression analysis, with factors set size (two to six), group (SZ vs HC), and time (early vs asymptotic trials; Fig. 2, top). Results showed main effects of all three factors (all $p$ values $<10^{-4}$ ) and interactions of set size with group and with time $(p=0.005$ and $p=$ 0.04 , respectively) but no interaction of group with time $(p=$ 0.95 ) or three-way interaction. We thus performed a separate analysis within early trials and within asymptotic trials. In early trials, we observed main effects of group and set size (both $p$ values $\left.<10^{-4}\right)$, indicating lower performance for patients and higher set sizes, but no interaction. In contrast, in late trials, both main effects $\left(p\right.$ values $<10^{-4}$ ) were qualified by interactions $(p=0.01)$, indicating that the effect of set size was larger for the SZ group.

We also investigated reaction times across the same factors (figure 2, bottom). There was a main effect of set size, indicating slower reaction times for higher set sizes $\left(p<10^{-4}\right)$; a main effect of time, indicating slower reaction times at the beginning of a learning block $\left(p<10^{-4}\right)$; but no main effect of group, indicating that controls and patients were, on average, as fast to respond $(p=0.11)$. However, there was an interaction of group with set size, indicating a stronger effect of set size on healthy controls $(p=0.006)$, and an interaction of group with time, indicating a stronger effect of learning on healthy controls $(p=$ 0.002 ). Specifically, patients' reaction times were less sensitive to difficulty or learning. However, restricting the analysis to the SZ group, we found a main effect of set size and time (both $p$ values $<10^{-3}$ ), indicating that their reaction times were sensitive to those factors, even if less so than controls and without interaction $(p=0.5)$. In contrast, within the HC group, we observed both main effects (both $p$ values $\left.<10^{-4}\right)$, as well as an interaction $(p=$ 0.02 ), showing that the effect of set size decreased with time.

In these analyses, set size is confounded with delay effects: indeed, each stimulus is seen, on average, every $1 / n_{\mathrm{S}}$ trials so that time-dependent forgetting could cause slower learning in higher set sizes, independently of load effects. However, on a trial-bytrial basis, these effects are dissociable: since stimulus order within each block was randomized, we can make use of the variability of delay between repetitions of a single stimulus to disentangle set-size and delay effects. We thus performed for each subject a logistic regression including three factors (inverse of set size, number of trials since last correct choice for the current 

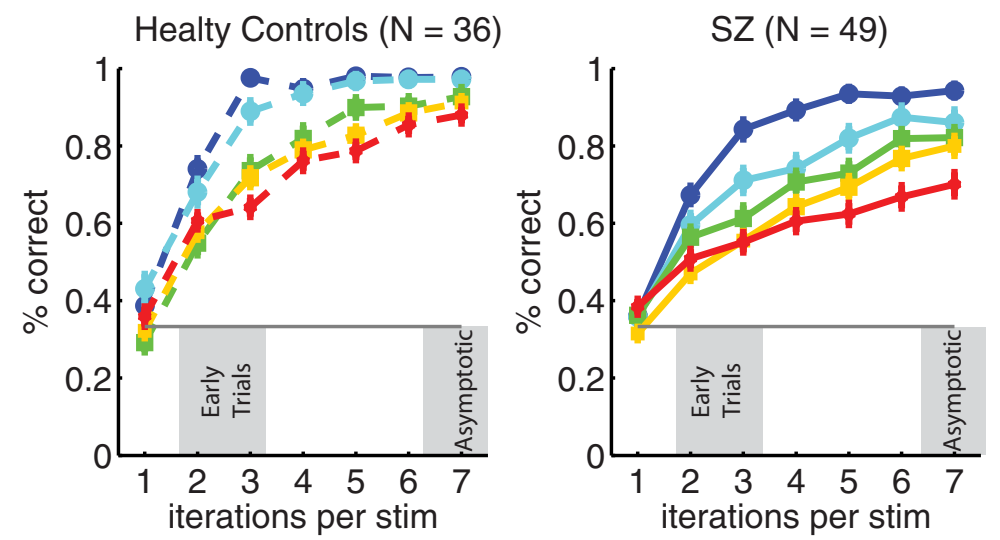

Set Size:

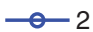

$\rightarrow-3$

$-4-4$

$-1=5$

$\rightarrow-6$

Groups:

$-\mathrm{SZ}$

$--\mathrm{HC}$

Early Trials

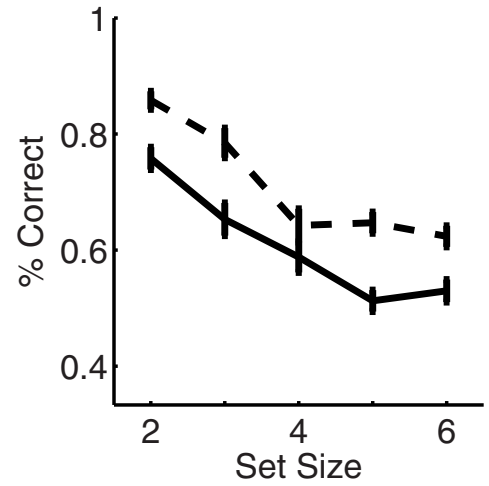

Asymptotic

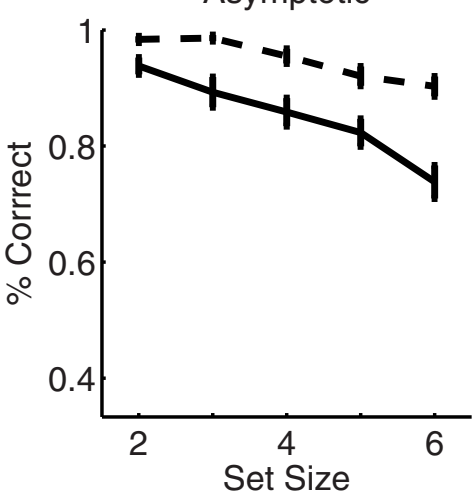

Overall Perf

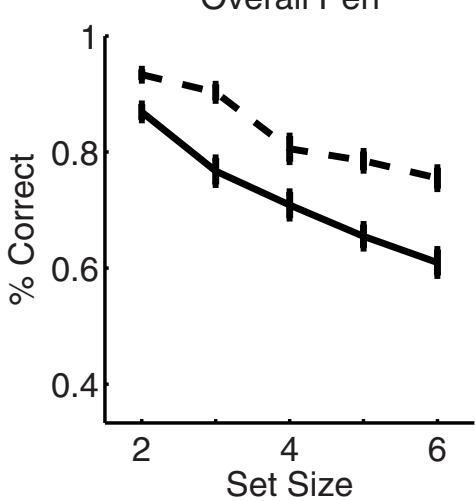

Early Trials

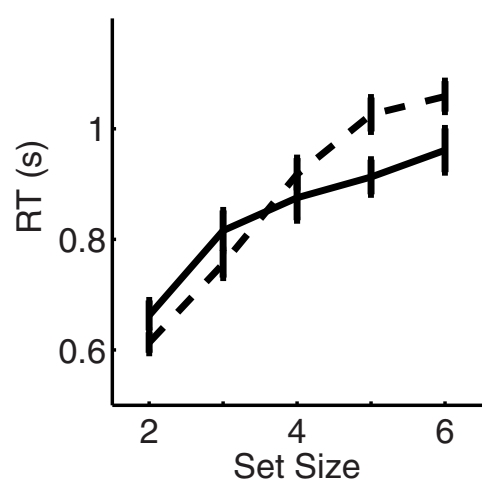

Asymptotic

Overall
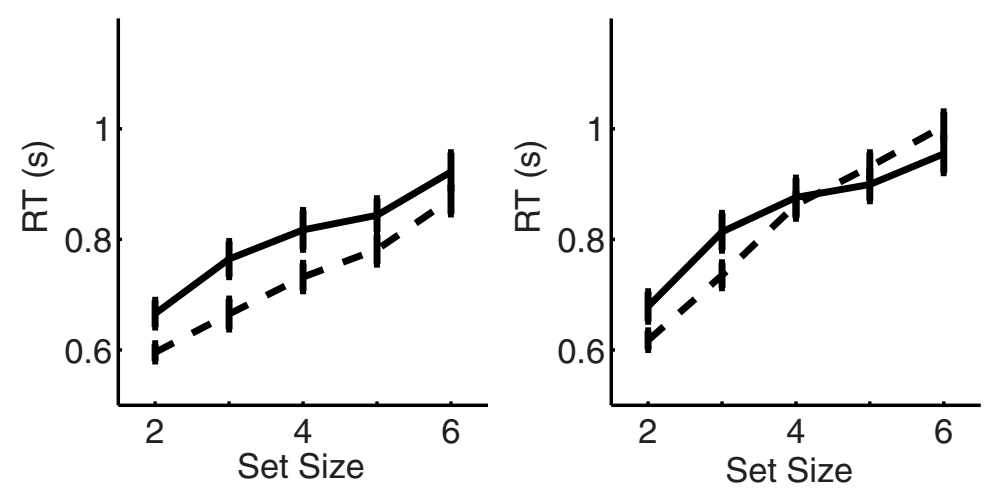

Figure 2. Learning effects. Top, Learning curves per set size and group (SZ, patients; HC, healthy controls, dashed lines). Learning curves indicate the probability of a correct response at the $n$th presentation of a single stimulus (stim). Middle, Average performance for early trials (presentations 2 and 3 of each stimulus), for asymptotic trials (last 2 presentations of each stimulus), and over the whole block. Bottom, Reaction times (RT) for early trials, asymptotic trials, and the whole block. Error bars indicate SEM.

stimulus, and number of previous correct choices for current stimulus) and analyzed regression weights across subjects. We hypothesized that set-size and delay factors should be indicative of a capacity-limited working memory process, whereas iteration should be representative of a RL-like slow, cumulative learning process.

Regression weights across the whole group were significantly nonzero for all three main effects (binomial test, all $p$ values $\left.<10^{-4}\right)$, as well as set-size interaction with delay $\left(p<10^{-4}\right)$ and with correct iterations $(p=0.002)$ and delay interaction with correct iterations $(p<0.05)$. Although set-size and iteration effects confirmed previous analysis indicating worse performance for higher set sizes and learning effects, delay effect showed that subjects were more likely to make an error when more trials intervened before a stimulus from a successful trial was presented again. Positive interaction of set size with delay indicated that this was more pronounced in higher load conditions, whereas negative interaction of delay with iterations indicated that the effect of delay decreased over time.

The previous effects all remained true when considering only the SZ group (all $p$ values $<0.05$ ), indicating that similarly to controls, patients' learning was determined not exclusively by feedback accumulation over time (as predicted by a single RL process), but also by set size and delay, hinting at their concurrent use of capacity-working memory to learn the associations. Logistic regression weights differed significantly between control and patients groups only for the fixed-effect $\beta$ weight $\left(t_{(83)}=4.7 ; p<\right.$ $\left.10^{-4}\right)$ and the set-size $\beta$ weight $\left(t_{(83)}=2.7 ; p=0.008\right)$, indicating a stronger effect of set size in SZ. No other weights differed ( $p$ values $>0.12$ ), and in particular, there was no difference between 

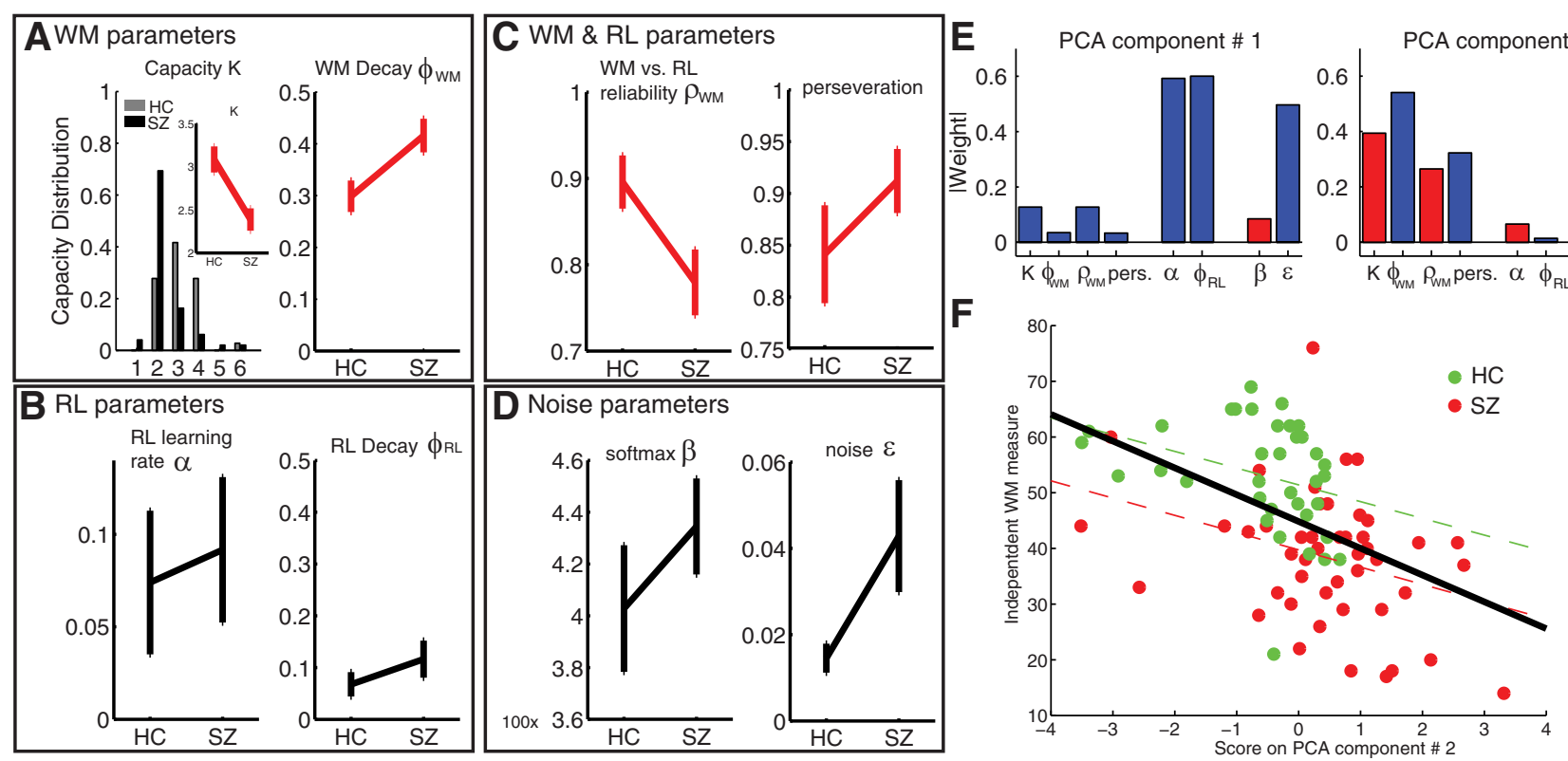

Figure 3. RLWM model fit parameters. Parameters fit on subjects' behavior with the RLWM model, for the HC group and patients (SZ). Red indicates a significant difference between groups. Error bars indicate SEM. A, WM-specific parameter rates show less robust WM for patients (lower capacity $N$ and faster decay rate $\phi_{\mathrm{WM}}$ ). $\boldsymbol{B}$, RL-specific parameters (learning rate $\alpha$ and decay $\phi_{\mathrm{RL}}$ ) show no difference. $\boldsymbol{C}$, Patients rely a priori less on WM $\left(\rho_{\mathrm{WM}}\right)$ and perseverate more than controls. $\boldsymbol{D}$, Despite worse performance in patients, noise parameters show no difference between groups, indicating that this is attributable to deficit in learning more than deficit in choice. $\boldsymbol{E}$, Absolute value of the weights on model parameters for the first two principal components. Blue bars indicate a positive weight and red bars a negative weight. The first component loads strongly on the two RL parameters, whereas the second component loads strongly on the four WM parameters. pers., Perseverate. $\boldsymbol{F}$, The second component is correlated with an independent classic measure of working memory across all subjects (black regression line) and within each group.

the groups in the effect of iteration number. These results are consistent with previous evidence suggestive of a role for WM in RL deficits in SZ. To separate more explicitly the effects of working memory, we fit subjects' data with reinforcement learning models.

Model fitting and model comparisons (see Materials and Methods) confirmed that subjects' learning was best represented by a mixture of two separate processes, one learning slowly and incrementally for all stimuli and the other storing information rapidly but with capacity limitations.

We next investigated the values of parameters obtained to confirm that they produced values for which the model was valid. First, the model fitting was successful in recovering two distinct processes. Indeed, across all subjects, the mean fit learning rate for the RL process was 0.084 (SD, 0.25) compared with the fixed learning rate of 1 for the WM memory process (Fig. 3). The mean fit decay rate for the RL process was 0.096 (SD, 0.21) compared with $0.37(\mathrm{SD}, 0.21)$ for the WM process (binomial test, $p=$ $\left.10^{-11}\right)$. This shows that the two modules had distinct learning dynamics, slow accumulation and nearly no forgetting for RL and fast learning but stronger forgetting for WM. Furthermore, both processes were indeed used: average WM reliability was $\sim 0.83$ $+/-0.24$, showing that it was efficiently used when a stimulus was stored, but capacity was found mostly within the two to four range (consistent with the WM literature), showing that RL was increasingly used to compensate for increasing failure of WM in higher set sizes when capacity was exceeded. Second, it is interesting to note that negative feedback was strongly neglected, as indicated by the high value of the perseveration parameter. Figure 4 shows simulation of the RLWM model with fitted parameters, as well as with the best-fitting pure RL models (see Material and Methods). This indicates that although both models can account for the qualitative effect of slower learning curves for higher set sizes, RLWM captures much better the learning dynamics exhibited in different sessions for each group.
We have shown that the recovered parameters are reasonable within the model hypothesis and that the fit model can adequately account for observed behavior. This allowed us next to use the model to better understand subjects' individual behaviors by summarizing individual differences into meaningful model parameters and comparing them across groups. Since most fit parameter distributions were non-normally distributed (see Materials and Methods), we compared results across groups with a nonparametric unpaired test (Wilcoxon rank sum test). We found (Fig. $3 A$ ) that both working memory-specific parameters were impaired in SZ compared with HC: patients showed lower working memory capacity (median of two vs three for controls; $\left.p<10^{-4}\right)$ and more forgetting in WM ( $\left.\phi_{\mathrm{WM}}, p=0.005\right)$. Furthermore, the reliance on WM use was lower in SZ (Fig. $3 C ; \rho_{\mathrm{WM}}$, $p=0.04)$. The parameter accounting for neglect of error information (or perseveration) in both WM and RL was significantly stronger in patients than controls (Fig. $3 C$; pers, $p=0.001$ ). In contrast, RL-specific parameters showed no difference between groups (Fig. $4 B ; \alpha, \phi_{\mathrm{RL}}, p>0.3$ ). The same was true of the noise parameter, $\beta$ (Fig. $3 D ; p=0.25$ ), though undirected noise was marginally higher for patients $(\varepsilon, p=0.06)$.

To investigate further the role of the different processes summarized by the fit model parameters, we performed a principal component analysis (PCA) on the $z$-scored fit parameters. We investigated the first two components, which accounted for $52 \%$ of the variance, and compared them to the MCCB working memory domain measure as an established measure of working memory performance. The first component loaded only on RL parameters (Fig. $3 E$ ) and undirected noise, did not differ significantly between groups $(p=0.49 ; t=0.69)$, and did not relate to a direct measure of WM (see Materials and Methods; $\rho=0.13$; $p=0.21$ ). On the contrary, the second component loaded on all WM parameters (Fig. 3E) and noise parameters, with higher value corresponding to lower capacity, higher forgetting, less WM use, and more perseveration. This encompasses all aspects of 
Empirical Data
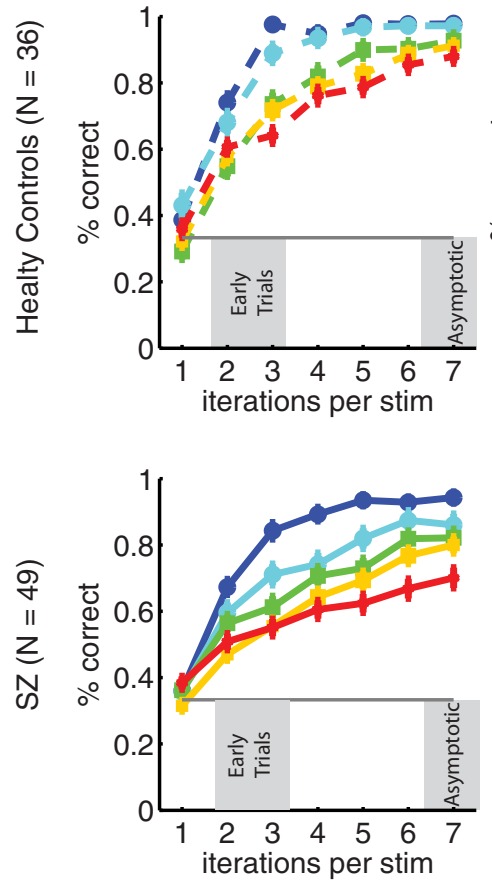

RLWM model simulation
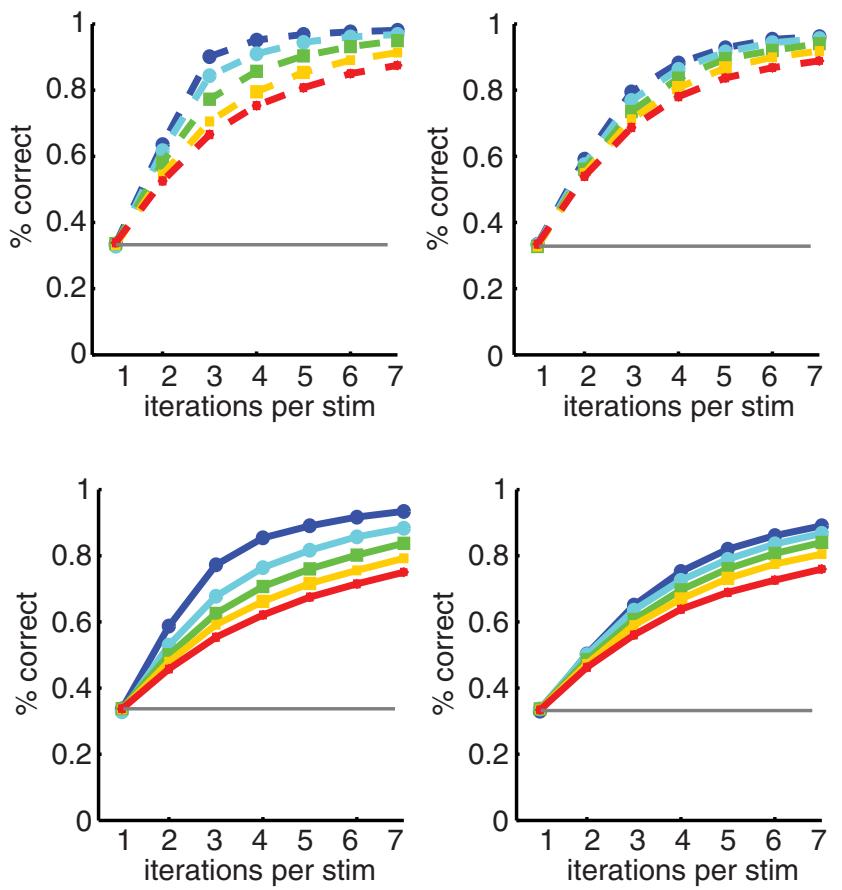

$\mathrm{RL}$ model simulation

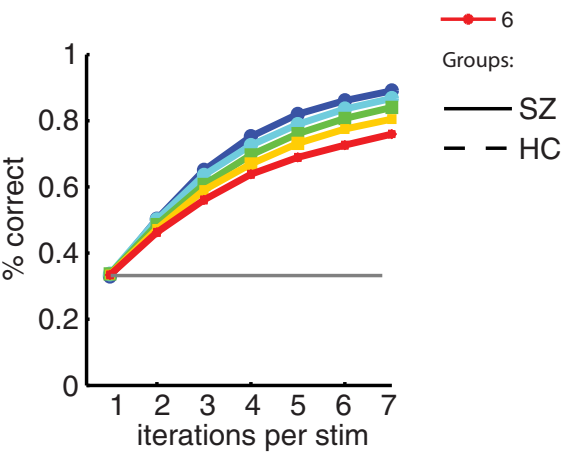

Figure 4. Learning curves and model simulations. Left, Empirical data per group (SZ, patients; HC, healthy controls, dashed lines). Middle, RLWM model simulation. Right, RL model simulation ( $\delta$ rule learning model including undirected noise, forgetting, and perseveration mechanisms). Learning curves indicate the probability of a correct response at the $n$th presentation of a single stimulus (stim). For each subject's fitted parameters, one set of learning curves was obtained by averaging over 100 simulations of the model with those parameters. Overall learning curves were then obtained by averaging across subjects.

the WM process, and thus, as expected, SZ subjects had a significantly higher score than controls (Fig. $3 F ; t=3.8 ; p=0.0003$ ). Notably, this score was significantly negatively correlated with the direct WM measure $\left(\rho=-0.52 ; p<10^{-6}\right)$, and this remained true within each group (both $p$ values $<0.05 ; \rho<-0.29$ ).

These results confirmed our hypothesis that the observed learning impairment in patients was related to a deficit in the working memory component of the model, rather than in reinforcement learning or in choice selection. We next investigated whether positive or negative symptoms of schizophrenia impacted any aspect of behavior. Based on prior work (Waltz et al., 2007, 2011), we had hypothesized that negative symptom severity might be related to the WM parameters. Correlations between symptoms and fit model parameters, as well as PCA components or the MCCB working memory measure, yielded no significant associations (BPRS, BNSS, and SANS scales; all $p$ values $>0.1$; Spearman $\rho<0.24)$. This lack of association with negative symptoms might reflect the fact that the current task more closely relates to the sort of rule-based working memory process that would involve lateral prefrontal cortex, whereas prior associations with negative symptoms involved representing specific reward values for each stimulus or action, functions attributed to more limbic portions of prefrontal cortex hypothesized to be related to negative symptoms, such as orbitofrontal cortex (Gold et al., 2012).

We also investigated the effects of drug dose on performance. We found a significant correlation between a Haloperidol equivalent dose and RL learning rate parameter $\alpha_{\mathrm{RL}}$ (Spearman $\rho=-$ $0.37 ; p=0.009$ ) as well as error neglect parameter pers (Spearman $\rho=0.46 ; p=0.001$ ), indicating more error neglect and slower learning for higher antipsychotic doses. Importantly, there was no effect on the working memory capacity parameter $(p=0.97)$ or any other parameter (all $p$ values $>0.11$ ). Finally, we found no effect of duration of illness on any parameters.

\section{Discussion}

These behavioral and modeling analyses provide a new perspective on the genesis of reinforcement learning deficits in people with schizophrenia. Our basic behavioral result that patients are overall less able than controls to use feedback to guide optimal response selection is consistent with many other reports in the literature (Waltz and Gold, 2007; Polli et al., 2008; Murray et al., 2008b; Somlai et al., 2011). Indeed, this body of behavioral work, combined with functional neuroimaging evidence suggesting abnormalities in the signaling of prediction errors in both cortical (Corlett et al., 2007) and striatal (Murray et al., 2008a; Koch et al., 2010) regions and abnormal striatal dopamine (Abi-Dargham et al., 1998; Howes et al., 2012), has led to a renewed focus on the role of reward-related dopaminergic mechanisms in both the positive and negative symptoms of schizophrenia (Kapur et al., 2005; Howes and Kapur, 2009; Ziauddeen and Murray, 2010; Deserno et al., 2013).

Our behavioral results and modeling strategy indicate, however, that learning impairments in SZ stem primarily from a core deficit in working memory, which may be responsible for what appears to be a reinforcement learning impairment. Specifically, we found robust evidence that patients had reduced working memory capacity, as well as faster working memory decay, coupled with normal reinforcement learning rates. This was observable in terms of a stronger detrimental effect of set size on learning in patients than in controls, together with a diminished effect of set size on reaction time. Assuming that the increased reaction time with set size arises from greater taxing of WM processes, the latter result is convergent with the modeling finding, 
not only of WM capacity and forgetting limitations, but also of less overall reliance on WM, among patients (even with their limited capacity). In contrast, the slow, incremental learning that is thought to be driven by dopaminergic prediction error signaling appears to be intact in schizophrenia, consistent with several recent reports from our group (Gold et al., 2012).

Note that we are not suggesting that reinforcement learning as a behavioral construct is normal in schizophrenia; the evidence for a behavioral deficit is clear (Waltz and Gold, 2007; Polli et al., 2008; Murray et al., 2008b; Somlai et al., 2011). Patients fail to learn from outcomes, and thus, functionally, patients show failures in reinforcement learning. This learning impairment, however, appears to arise primarily as a consequence of WM deficits.

This conclusion was substantially aided by the use of computational modeling to estimate contributions of distinct processes. Much as it did for healthy participants (Collins and Frank, 2012), model fitting revealed that behavior is better accounted for by a mixture of WM and RL processes than by single process models, for both controls and patients. Moreover, our prior work showed that two of the key parameters characterizing WM and RL systems are related to genetic variations in prefrontal versus striatal function, respectively. The use of computational modeling for teasing apart the purported neural and psychological processes governing motivational deficits in learning and decision making may be particularly useful with disorders, such as schizophrenia, that involve multiple cognitive deficits (Montague et al., 2012; Wiecki et al., 2014). Indeed, it is difficult to isolate the role of specific processes in a quantifiable fashion using behavioral or imaging methods alone. Computational modeling methods, however, provide a method to parse out different contributions to behavior.

It is interesting to note that the patient group did not differ from controls in noise parameters. This is important evidence that the patients did not simply have a noisier decision strategy in the face of a challenging task: their impairment is in learning the associations, not in using the learned associations to make a decision.

Deficits in working memory have long been considered a central feature of schizophrenia (Lee and Park, 2005; Barch et al., 2009). Indeed, one reason working memory has been such a focus of the schizophrenia literature is that it is a critical resource for many other aspects of cognition, ranging from fluid reasoning to language comprehension (Just and Carpenter, 1992; Johnson et al., 2013). Thus, working memory impairments could reasonably provide a principled account of many of the cognitive deficits characteristic of schizophrenia. Here, we show that this same impairment has consequences for reinforcement learning, thereby having broad implications for motivational processes.

A potential limitation of our study is that, contrary to many published studies, our experiment uses deterministic rather than probabilistic reinforcement feedback. Although this allows more straightforward interpretation and modeling of the potential content of working memory, it may weaken the sensitivity to the contribution of "RL" mechanisms to overall performance: in the absence of probabilistic feedback, prediction errors are less variable over time and the integration process of RL less critical. In principle, probabilistic tasks may be more sensitive to gradual RL mechanisms, whereas WM mechanisms may be more useful with deterministic feedback. Thus, we do not mean to suggest that our account of the data from this experiment can be confidently generalized to the larger clinical literature. However, it is interesting to note that patients sometimes demonstrate more severe learning impairments on nearly deterministic stimulus pairs (e.g., in the case of $90 \%$ validly reinforced responses, which, in principle, should be easier to learn), but more "normal" performance on less deterministic pairs (e.g., in the case of $80 \%$ validly reinforced responses, which are more slowly acquired; Gold et al., 2012). This suggests a role for WM in some probabilistic learning environments (Kim et al., 2007). Thus, we suspect that WM impairments may well play an important and previously unknown role in the learning, decision making, and motivational deficits observed in people with schizophrenia.

Several other results deserve discussion. First, we found that both patients and controls tended to neglect errors but that patients were more likely to do so and persist with prior response choices. This is consistent with numerous prior reports of perseveration in the schizophrenia literature, beginning with studies using the Wisconsin Card Sorting Test (Goldberg, 1987; Prentice et al., 2008). Interestingly, there are now multiple reports of relatively normal feedback error-related negativity in schizophrenia (Morris et al., 2011; Horan et al., 2012), suggesting that the error information is processed but fails to fully impact behavior.

Second, model fits showed that patients relied less on the working memory process relative to RL process, as indicated by a significantly lower reliability parameter, $\rho_{\mathrm{WM}}$. One possible interpretation is that storage of any individual stimulus into working memory is less reliable (even within capacity) and is thus less likely to be used by patients, highlighting another limitation of their working memory system. However, another interpretation is in the arbitration between the use of the WM and RL systems. Indeed, the WM system is capacity limited, so that if subjects completely rely on it, their best performance will be constrained by capacity. But they could also decide to not rely on WM as much even if within their capacity, e.g., because they have learned over the course of their life that WM is not likely to be successful in producing the right behaviors. This could arise because capacity is lower and thus more often unhelpful, or because of more noise within WM even when under capacity. Thus, they might generalize that to all situations regardless of whether that situation could actually be solved within their capacity. Although the current results cannot distinguish between those two possibilities, they both support the interpretation of impaired working memory as a source of slower learning.

Finally, as noted above, we found a negative relationship between antipsychotic dose and RL learning rate. Whereas it is tempting to conclude that substantial blockade of striatal dopamine receptors is responsible for this association, this result should be regarded with caution. Recall that the patients and controls did not differ on RL learning rate, and that all of the patients were taking antipsychotic medications. Thus, if antipsychotics had a dose-dependent effect on RL, one would expect robust between-group differences. Furthermore, close examination of the data revealed that the performance correlations were primarily driven by a small number of patients who were taking very high doses of antipsychotics $(>20 \mathrm{mg}$ of haloperidol equivalents). Thus, this association could exist either because a high degree of $\mathrm{D}_{2}$ blockade is needed to "overcompensate" for excess striatal DA levels, and thus impair RL, or it could also simply reflect the possibility that those patients taking very high doses were the most ill and least treatment responsive. In the absence of random assignment to antipsychotic drug, the interpretation of drug dose correlations with behavioral or modeling parameters is necessarily confounded. However, the notion that very high doses of antipsychotic medication might interfere with RL is clearly plausible (Beninger et al., 2003). Indeed, it would be somewhat surprising if this were not the case. 
In summary, through the use of a novel behavioral task and computational model, it appears that working memory impairments may be critically implicated in the reinforcement learning deficits found in schizophrenia. It remains for future work to determine whether this understanding generalizes to other types of learning and decision-making tasks.

\section{References}

Abi-Dargham A, Gil R, Krystal J, Baldwin RM, Seibyl JP, Bowers M, van Dyck CH, Charney DS, Innis RB, Laruelle M (1998) Increased striatal dopamine transmission in schizophrenia: confirmation in a second cohort. Am J Psychiatry 155:761-767. Medline

American Psychiatric Association (2000) DSM-IV-TR: diagnostic and statistical manual of mental disorders, text revision. Washington, DC: American Psychiatric Associations.

Andreasen N (1983) Scale for the assessment of negative symptoms. srspence.com. Available at http://www.srspence.com/SANS.pdf. Retrieved January 17, 2014.

Barch DM, Ceaser A (2012) Cognition in schizophrenia: core psychological and neural mechanisms. Trends Cogn Sci 16:27-34. CrossRef Medline

Barch DM, Berman MG, Engle R, Jones JH, Jonides J, Macdonald A 3rd, Nee DE, Redick TS, Sponheim SR (2009) CNTRICS final task selection: working memory. Schizophr Bull 35:136-152. CrossRef Medline

Beninger RJ, Wasserman J, Zanibbi K, Charbonneau D, Mangels J, Beninger BV (2003) Typical and atypical antipsychotic medications differentially affect two nondeclarative memory tasks in schizophrenic patients: a double dissociation. Schizophr Res 61:281-292. CrossRef Medline

Burnham KP, Anderson DR (2002) Model selection and multi-model inference: a practical information-theoretic approach. Springer, New York. E-book available at http://www.citeulike.org/group/7954/article/4425594.

Collins AG, Frank MJ (2012) How much of reinforcement learning is working memory, not reinforcement learning? A behavioral, computational, and neurogenetic analysis. Eur J Neurosci 35:1024-1035. CrossRef Medline

Collins A, Koechlin E (2012) Reasoning, learning, and creativity: frontal lobe function and human decision-making. PLoS Biol 10:e1001293. CrossRef Medline

Collins AG, Frank MJ (2013) Cognitive control over learning: creating, clustering, and generalizing task-set structure. Psychol Rev 120:190-229. CrossRef Medline

Corlett PR, Murray GK, Honey GD, Aitken MR, Shanks DR, Robbins TW, Bullmore ET, Dickinson A, Fletcher PC (2007) Disrupted predictionerror signal in psychosis: evidence for an associative account of delusions. Brain 130:2387-2400. CrossRef Medline

Daw N (2011) Trial-by-trial data analysis using computational models. In: Decision making, affect, and learning: attention and performance XXIII (Delgado MR, Phelps EA, Robbins TW, eds), 1-26. New York: Oxford UP.

Dayan P, Daw ND (2008) Decision theory, reinforcement learning, and the brain. Cogn Affect Behav Neurosci 8:429-453. CrossRef Medline

Deserno L, Boehme R, Heinz A, Schlagenhauf F (2013) Reinforcement learning and dopamine in schizophrenia: dimensions of symptoms or specific features of a disease group? Front Psychiatry 4:172. CrossRef Medline

Doll BB, et al. (2014) Reduced susceptibility to confirmation bias in schizophrenia. Cogn Affect Behav Neurosci 14:715-728. CrossRef Medline

Dowd EC, Barch DM (2012) Pavlovian reward prediction and receipt in schizophrenia: relationship to anhedonia. PloS One 7:e35622. CrossRef Medline

First MB, Spitzer RL, Gibbon M, Williams JB (1997) Structured clinical interview for DSM-IV axis I disorders, clinician version (SCID-CV). Washington, DC: American Psychiatric Association.

Frank MJ, Seeberger LC, O'reilly RC (2004) By carrot or by stick: cognitive reinforcement learning in parkinsonism. Science 306:1940-1943. CrossRef Medline

Frank MJ, Moustafa AA, Haughey HM, Curran T, Hutchison KE (2007) Genetic triple dissociation reveals multiple roles for dopamine in reinforcement learning. Proc Natl Acad Sci U S A 104:16311-16316. CrossRef Medline

Gogos JA, Morgan M, Luine V, Santha M, Ogawa S, Pfaff D, Karayiorgou M (1998) Catechol-O-methyltransferase-deficient mice exhibit sexually di- morphic changes in catecholamine levels and behavior. Proc Natl Acad Sci U S A 95:9991-9996. CrossRef Medline

Gold JM, Waltz JA, Prentice KJ, Morris SE, Heerey EA (2008) Reward processing in schizophrenia: a deficit in the representation of value. Schizophr Bull 34:835-847. CrossRef Medline

Gold JM, Waltz JA, Matveeva TM, Kasanova Z, Strauss GP, Herbener ES, Collins AG, Frank MJ (2012) Negative symptoms and the failure to represent the expected reward value of actions: behavioral and computational modeling evidence. Control 69:129-138. CrossRef Medline

Goldberg TE, Weinberger DR, Berman KF, Pliskin NH, Podd MH (1987) Further evidence for dementia of the prefrontal type in schizophrenia? Arch Gen Psychiatry 44:1008-1014. CrossRef Medline

Guitart-Masip M, Huys QJ, Fuentemilla L, Dayan P, Duzel E, Dolan RJ (2012) Go and no-go learning in reward and punishment: interactions between affect and effect. Neuroimage 62:154-166. CrossRef Medline

Horan WP, Foti D, Hajcak G, Wynn JK, Green MF (2012) Impaired neural response to internal but not external feedback in schizophrenia. Psychol Med 42:1637-1647. CrossRef Medline

Howes OD, Kapur S (2009) The dopamine hypothesis of schizophrenia: version III-the final common pathway. Schizophr Bull 35:549-562. CrossRef Medline

Howes OD, Kambeitz J, Kim E, Stahl D, Slifstein M, Abi-Dargham A, Kapur S (2012) The nature of dopamine dysfunction in schizophrenia and what this means for treatment. Arch Gen Psychiatry 69:776-786. CrossRef Medline

Huotari M, Gogos JA, Karayiorgou M, Koponen O, Forsberg M, Raasmaja A, Hyttinen J, Männistö PT (2002) Brain catecholamine metabolism in catechol-O-methyltransferase (COMT)-deficient mice. Eur J Neurosci 15:246-256. CrossRef Medline

Johnson MK, McMahon RP, Robinson BM, Harvey AN, Hahn B, Leonard CJ, Luck SJ, Gold JM (2013) The relationship between working memory capacity and broad measures of cognitive ability in healthy adults and people with schizophrenia. Neuropsychology 27:220-229. CrossRef Medline

Just MA, Carpenter PA (1992) A capacity theory of comprehension: individual differences in working memory. Psychol Rev 99:122-149. CrossRef Medline

Kapur S, Mizrahi R, Li M (2005) From dopamine to salience to psychosislinking biology, pharmacology and phenomenology of psychosis. Schizophr Res 79:59-68. CrossRef Medline

Kim H, Lee D, Shin YM, Chey J (2007) Impaired strategic decision making in schizophrenia. Brain Res 1180:90-100. CrossRef Medline

Kirkpatrick B, Strauss GP, Nguyen L, Fischer BA, Daniel DG, Cienfuegos A, Marder SR (2011) The brief negative symptom scale. psychometric properties. Schizophr Bull 37:300-305. CrossRef Medline

Koch K, Schachtzabel C, Wagner G, Schikora J, Schultz C, Reichenbach JR, Sauer H, Schlösser RG (2010) Altered activation in association with reward-related trial-and-error learning in patients with schizophrenia. Neuroimage 50:223-232. CrossRef Medline

Lee J, Park S (2005) Working memory impairments in schizophrenia: a meta-analysis. J Abnormal Psychol 114:599-611. CrossRef Medline

Lobo MK, Cui Y, Ostlund SB, Balleine BW, Yang XW (2007) Genetic control of instrumental conditioning by striatopallidal neuron-specific S1P receptor Gpr6. Nat Neurosci 10:1395-1397. CrossRef Medline

Matsumoto M, Weickert CS, Akil M, Lipska BK, Hyde TM, Herman MM, Kleinman JE, Weinberger DR (2003) Catechol O-methyltransferase mRNA expression in human and rat brain: evidence for a role in cortical neuronal function. Neuroscience 116:127-137. CrossRef Medline

Montague PR, Dolan RJ, Friston KJ, Dayan P (2012) Computational psychiatry. Trends Cogn Sci 16:72-80. CrossRef Medline

Morris SE, Holroyd CB, Mann-Wrobel MC, Gold JM (2011) Dissociation of response and feedback negativity in schizophrenia: electrophysiologi$\mathrm{cal}$ and computational evidence for a deficit in the representation of value. Front Hum Neurosci 5:123. CrossRef Medline

Murray GK, Corlett PR, Clark L, Pessiglione M, Blackwell AD, Honey G, Jones PB, Bullmore ET, Robbins TW, Fletcher PC (2008a) Substantia nigra/ventral tegmental reward prediction error disruption in psychosis. Mol Psychiatry 13:267-276. CrossRef Medline

Murray GK, Cheng F, Clark L, Barnett JH, Blackwell AD, Fletcher PC, Robbins TW, Bullmore ET, Jones PB (2008b) Reinforcement and reversal learning in first-episode psychosis. Schizophr Bull 34:848-855. CrossRef Medline 
Nuechterlein K, Green M (2006) MCCB: matrics consensus cognitive battery. Los Angeles: MATRICS Assessment.

O’Doherty JP, Hampton A, Kim H (2007) Model-based fMRI and its application to reward learning and decision making. Ann N Y Acad Sci 1104: 35-53. CrossRef Medline

Overall JE, Gorham DR (1962) The brief psychiatric rating scale. Psychol Rep 10:799-812. CrossRef

Paulus MP, Frank L, Brown GG, Braff DL (2003) Schizophrenia subjects show intact success-related neural activation but impaired uncertainty processing during decision-making. Neuropsychopharmacology 28:795806. CrossRef Medline

Petrides M (1985) Deficits on conditional associative-learning tasks after frontal- and temporal-lobe lesions in man. Neuropsychologia 23:601614. CrossRef Medline

Pfohl B, Blum N, Zimmerman M (1997) Structured interview for DSM-IV personality. Washington, DC: American Psychiatric Publishing.

Polli FE, Barton JJ, Thakkar KN, Greve DN, Goff DC, Rauch SL, Manoach DS (2008) Reduced error-related activation in two anterior cingulate circuits is related to impaired performance in schizophrenia. Brain 131:971986. CrossRef Medline

Prentice KJ, Gold JM, Buchanan RW (2008) The Wisconsin Card Sorting impairment in schizophrenia is evident in the first four trials. Schizophr Res 106:81-87. CrossRef Medline

Schlagenhauf F, Huys QJ, Deserno L, Rapp MA, Beck A, Heinze HJ, Dolan R, Heinz A (2013) Striatal dysfunction during reversal learning in unmedicated schizophrenia patients. Neuroimage 89:171-180. CrossRef Medline

Schwarz G (1978) Estimating the dimension of a model. Ann Stat 6:461464. CrossRef

Shurman B, Horan WP, Nuechterlein KH (2005) Schizophrenia patients demonstrate a distinctive pattern of decision-making impairment on the Iowa Gambling Task. Schizophr Res 72:215-224. CrossRef Medline

Slifstein M, Kolachana B, Simpson EH, Tabares P, Cheng B, Duvall M, Frankle WG, Weinberger DR, Laruelle M, Abi-Dargham A (2008) COMT genotype predicts cortical-limbic D1 receptor availability mea- sured with [11C]NNC112 and PET. Mol Psychiatry 13:821-827. CrossRef Medline

Somlai Z, Moustafa AA, Kéri S, Myers CE, Gluck MA (2011) General functioning predicts reward and punishment learning in schizophrenia. Schizophr Res 127:131-136. CrossRef Medline

Stephan KE, Penny WD, Daunizeau J, Moran RJ, Friston KJ (2009) Bayesian model selection for group studies. Neuroimage 46:1004-1017. CrossRef Medline

Strauss GP, Robinson BM, Waltz JA, Frank MJ, Kasanova Z, Herbener ES, Gold JM (2011) Patients with schizophrenia demonstrate inconsistent preference judgments for affective and nonaffective stimuli. Schizophr Bull 37:1295-1304. CrossRef Medline

Waltz JA, Gold JM (2007) Probabilistic reversal learning impairments in schizophrenia: further evidence of orbitofrontal dysfunction. Schizophr Res 93:296-303. CrossRef Medline

Waltz JA, Frank MJ, Robinson BM, Gold JM (2007) Selective reinforcement learning deficits in schizophrenia support predictions from computational models of striatal-cortical dysfunction. Biol Psychiatry 62:756-764. CrossRef Medline

Waltz JA, Schweitzer JB, Gold JM, Kurup PK, Ross TJ, Salmeron BJ, Rose EJ, McClure SM, Stein EA (2009) Patients with schizophrenia have a reduced neural response to both unpredictable and predictable primary reinforcers. Neuropsychopharmacology 34:1567-1577. CrossRef Medline

Waltz JA, Frank MJ, Wiecki TV, Gold JM (2011) Altered probabilistic learning and response biases in schizophrenia: behavioral evidence and neurocomputational modeling. Neuropsychology 25:86-97. CrossRef Medline

Wechsler D (1999) WASI (Wechsler Adult Scale-reduced). New York: The Psychological Corporation.

Wiecki TV, Poland J, Frank MJ (2014) Model-based cognitive neuroscience approaches to computational psychiatry: clustering and classification. Clin Psychol Sci, in press.

Ziauddeen H, Murray GK (2010) The relevance of reward pathways for schizophrenia. Curr Opin Psychiatry 23:91-96. CrossRef Medline 